\title{
DIAGNÓSTICO Y SEGUIMIENTO DEL FETO CON RESTRICCIÓN DEL CRECIMIENTO INTRAUTERINO (RCIU) Y DEL FETO PEQUEÑO PARA LA EDAD GESTACIONAL (PEG). CONSENSO COLOMBIANO*
}

\author{
Diagnosing and managing foetuses suffering from intrauterine \\ growth restriction (IUGR) and foetuses which are small \\ for their gestational age (SGA). Colombian consensus \\ José Enrique Sanín-Blair, M.D.**,***, Jaime Gómez Díaz, M.D.***, \\ Jorge Ramírez, M.D.***, Carlos Alberto Mejía, M.D.***, Óscar Medina, M.D.***, \\ José Vélez, M.D. ***, Consenso Nacional de Expertos**** \\ Recibido: abril 12/09 - Aceptado: julio 31/09
}

\section{RESUMEN}

Objetivo: dar a conocer a los participantes en el cuidado de la salud de la mujer embarazada, los más recientes avances y recomendaciones clínicas de un tema controversial que es causa importante de morbimortalidad perinatal en Colombia.

Metodología: se revisaron las bases de datos Cochrane, Medline y Embase, además de la base latinoamericana SciELO, libros de la especialidad y consensos de otras sociedades científicas en búsqueda de estudios diagnósticos, revisiones sistemáticas, estudios aleatorizados y metaanálisis relativos a los términos: "restricción de crecimiento intrauterino" y “pequeño para la edad gestacional” en el período comprendido entre 1995-2009. A partir de esto, se realizó un manuscrito inicial, el cual fue sometido

* La parte inicial de este consenso fue presentada en el Simposio de RCIU, organizado por Medicina Fetal S.A. en Medellín (Colombia) en septiembre de 2008.

** Gineco-obstetra. Coordinador del Consenso Colombiano de RCIU. Especialista en Medicina Materno-Fetal. Clínica Universitaria Bolivariana, Medellín (Colombia). Correo electrónico: saninblair@ yahoo.com

*** Gineco-obstetra. Especialista en Ultrasonido. Medicina Fetal S.A, Medellín (Colombia).

**** Lista completa al final del documento. a discusión por medio de Internet. Posteriormente, el manuscrito final fue discutido y modificado en la ciudad de Medellín (Colombia), en el marco del congreso de Medicina Fetal S.A. en septiembre de 2008.

Resultados: teniendo en cuenta la evidencia disponible, se presentó una guía para el manejo del feto PEG y con RCIU, la cual puede ser aplicada a Colombia.

Conclusiones: este tema genera controversia debido a la falta de evidencia científica para la aplicación de algunas definiciones y pruebas diagnósticas en su manejo.

Palabras clave: pequeño para la edad gestacional, restricción de crecimiento intrauterino, ultrasonido, Doppler.

\section{SUMMARY}

Objective: making those participating in pregnant women's health care aware of the latest clinical recommendations regarding a controversial topic which is an important cause of perinatal morbidity in Colombia.

Methodology: the Cochrane Library, Medline and Embase databases were searched as well as 
SciELO Latin-American database, specialised books and consensus from other scientific societies for diagnostic studies, systematic reviews, randomised trials and meta-analysis carried out between 19952009 regarding the terms "intrauterine growth restriction" and "small for gestational age". An initial manuscript was written and submitted for on-line discussion. The final manuscript was discussed and amended in Medellín, Colombia, during the Foetal Medicine Congress held in September 2008.

Results: a guide to managing SGA and IUGR foetuses was written and is presented here; it has been based on the best available evidence and could be applied in Colombia.

Conclusions: this issue produces controversy due to a lack of scientific evidence for applying some definitions and diagnostic tests when managing IUGR and SGA foetuses.

Key words: small for gestational age, intrauterine growth restriction, ultrasound, Doppler.

\section{INTRODUCCIÓN Y JUSTIFICACIÓN DEL PROBLEMA}

La restricción del crecimiento intrauterino (RCIU) y el feto pequeño para la edad gestacional (PEG) se presentan en $4 \%$ a $15 \%$ de los embarazos. ${ }^{1-7}$ Esta variación tan grande en la frecuencia puede explicarse debido a las distintas definiciones utilizadas por los autores para hacer el diagnóstico, a las curvas de crecimiento fetal utilizadas para evaluar cada caso y a las diferencias entre las poblaciones estudiadas. ${ }^{1-7}$

No existen estadísticas en nuestro medio para saber con exactitud cuál es la prevalencia del déficit de crecimiento fetal en la población de gestantes embarazadas. Sin embargo, en el Departamento Administrativo Nacional de Estadística de Colombia (DANE) figuran registrados entre los años 2004, 2005 y 2006, 2.157.517 nacidos vivos, de los cuales 175.829 (8,1 \%) fueron catalogados como con peso inferior a 2.500 gramos o bajo peso al nacer. Este grupo de pacientes pertenece a partos pretérmino y a neonatos con alteraciones de crecimiento, lo cual no hace posible la diferenciación. No obstante, dentro de las causas de muerte fetal para los mismos años, el DANE registra 70.545 muertes fetales, de las cuales $1.494(2,1 \%)$ fueron catalogadas como debidas directamente al retardo del crecimiento fetal, a la desnutrición fetal, a la gestación corta y al bajo peso al nacer. Esto, sin tomar en cuenta que la RCIU es un estado comórbido y que sumado al aumento de la morbilidad perinatal y a largo plazo que presenta esta patología, justifica que se tomen medidas para la prevención primaria, secundaria y terciaria. $^{8}$

En las últimas décadas han aparecido múltiples definiciones, clasificaciones, guías de manejo, además de nuevas tecnologías de mayor o de menor utilidad para la evaluación de estos pacientes, generando amplia discusión y opiniones disímiles sobre algunas preguntas específicas acerca de esta enfermedad. Por consiguiente, el objetivo de este consenso es dar a conocer a los gineco-obstetras, especialistas en medicina materno-fetal, perinatólogos, residentes de la especialidad, médicos generales y todos los participantes en el cuidado de la salud de la mujer embarazada, las recomendaciones clínicas de un tema controversial que es causa importante de morbimortalidad perinatal en nuestro medio. De este modo, se hace énfasis en que la identificación y el manejo adecuado del feto con RCIU permiten disminuir las tasas de mortalidad y morbilidad fetal, y las complicaciones neonatales a corto y a largo plazo entre las cuales se incluyen: la prematuridad, la encefalopatía hipóxico-isquémica, la enterocolitis necrotizante, la hemorragia intraventricular, la aspiración de meconio, la policitemia, la hipoglucemia y las complicaciones metabólicas. De otro lado, la identificación de un feto pequeño para la edad gestacional, que no presente RCIU, permite disminuir las intervenciones obstétricas innecesarias y la morbilidad materna causada por éstas.

\section{MATERIALES Y MÉTODOS}

Se revisaron las bases de datos Cochrane, Medline y Embase, además de la base latinoamericana 
SciELO, libros de la especialidad y consensos de otras sociedades científicas en búsqueda de estudios diagnósticos, revisiones sistemáticas, estudios aleatorizados y análisis relativos a los términos: "intrauterine growth restriction" y "small for gestational age" (restricción de crecimiento intrauterino y pequeño para la edad gestacional) en el período comprendido entre 1995-2009. En total, se recopilaron 12.106 artículos, los cuales fueron clasificados por 3 revisores independientes quienes tenían conocimiento de epidemiología y medicina basada en evidencia. De acuerdo con criterios metodológicos, se seleccionaron los artículos a estudiar y finalmente se revisaron a texto completo 83 artículos médicos. Los niveles de evidencia y grados de recomendación fueron asignados a partir de la clasificación de Sacket ${ }^{9}$ (tabla 1) y según los criterios del Centro de Medicina Basada en la Evidencia (CEBM, por sus siglas en inglés). Posteriormente, se elaboró un manuscrito inicial y se invitó a los coordinadores de universidades con posgrado en Ginecología y Obstetricia y a los coordinadores de diferentes hospitales a hacer aportes en la elaboración del consenso. Para tal fin, este manuscrito fue publicado en la página de Internet diseñada para la realización del consenso (http://groups.google.com/group/consenso-rciu), donde además, los participantes del foro virtual de discusión sugerían modificaciones e ingresaban artículos para la elaboración de la guía, los cuales pueden ser consultados en dicha página. Finalmente, la última versión del manuscrito fue discutida y modificada en la ciudad de Medellín (Colombia) en el marco del congreso de Medicina Fetal S.A. en septiembre de 2008. Este encuentro de consenso fue considerado una reunión de expertos, en la cual sus participantes resaltaron que una guía constituye un elemento básico para la atención pero que es igualmente importante hacer énfasis en que cada paciente requiere una evaluación individualizada según su condición y teniendo en cuenta el nivel de complejidad y de recursos con que cuenta el médico especialista.

Este consenso brinda aclaración e información sobre las siguientes patologías registradas en la clasificación internacional de enfermedades (CIE). ${ }^{10}$

\section{Tabla 1. Niveles de evidencia de acuerdo con las recomendaciones de Sacket.}

\section{Nivel de evidencia:}

Ia: la evidencia proviene de metaanálisis de ensayos controlados, aleatorizados, bien diseñados.

Ib: la evidencia proviene de, al menos, un ensayo controlado aleatorizado.

IIa: la evidencia proviene de, al menos, un estudio controlado bien diseñado sin aleatorizar.

IIb: la evidencia proviene de, al menos, un estudio no completamente experimental, bien diseñado, como los estudios de cohortes. Se refiere a la situación en la que la aplicación de una intervención está fuera del control de los investigadores, pero su efecto puede evaluarse.

III: la evidencia proviene de estudios descriptivos no experimentales bien diseñados, como los estudios comparativos, estudios de correlación o estudios de casos y controles.

IV: la evidencia proviene de documentos u opiniones de comités de expertos o experiencias clínicas de autoridades de prestigio o los estudios de series de casos.

\section{Grado de la recomendación:}

A: basada en una categoría de evidencia I. Extremadamente recomendable.

B: basada en una categoría de evidencia II. Recomendación favorable.

C: basada en una categoría de evidencia III. Recomendación favorable pero no concluyente.

D: basada en una categoría de evidencia IV. Consenso de expertos, sin evidencia adecuada de investigación. 
- 0365 Atención materna por déficit del crecimiento fetal.

- PO 050 Bajo peso para la edad gestacional.

- PO 051 Pequeño para la edad gestacional.

- PO 052 Desnutrición fetal sin mención de peso o talla bajos para la edad gestacional.

- PO 059 Retardo del crecimiento fetal no especificado.

- 283 Hallazgo ultrasónico anormal en el examen prenatal de la madre.

\section{DEFINICIÓN Y CONSIDERACIONES}

En el período antenatal, la medición es indirecta y por lo tanto es más difícil y menos exacta que la antropometría neonatal. ${ }^{2,5,10}$ El único medio que permite de manera adecuada sospechar y diagnosticar las alteraciones en el crecimiento fetal es el ultrasonido. ${ }^{1-7}$ Para evaluar estas alteraciones, es necesario conocer la edad gestacional, calcular el peso fetal por ultrasonido y contar con curvas de crecimiento apropiadas para establecer los percentiles de crecimiento de cada feto. La opinión de este consenso es que se requieren datos de la población colombiana para tener una aproximación más real al problema, ya que los estudios existentes no permiten la masificación de su uso. ${ }^{11}$ Ahora bien, ante la ausencia de tablas validadas en nuestro medio, se recomienda emplear las tablas de Hadlock ${ }^{10}$ para cálculo de peso, perímetro abdominal, circunferencia fetal y fémur. De hecho, estudios poblacionales realizados en Chile ${ }^{12-14}$ y Uruguay, ${ }^{15}$ mostraron similitud con los parámetros biométricos publicados por Hadlock; asimismo, estas tablas están ampliamente difundidas y hacen parte del software de la mayoría de las máquinas de ultrasonido (nivel de evidencia III, recomendación tipo C).

De otra parte, según el consenso basado en las diferentes recomendaciones internacionales ${ }^{4-7,14,16}$ los fetos que presentan uno o varios de los siguientes parámetros son considerados como posibles casos de RCIU:
- Crecimiento fetal por debajo del percentil 10 para la edad gestacional con signos de compromiso fetal que incluyen anormalidades de la circulación feto placentaria identificadas por Doppler, disminución del líquido amniótico o alteraciones en las pruebas de bienestar fetal (perfil biofísico fetal, monitoreo no estresante - NST).

- El feto con un peso inferior al percentil 3 para la edad gestacional calculado por ecografía.

- Feto con circunferencia abdominal por debajo del percentil 2,5 para la edad gestacional sin alteraciones de otros parámetros biométricos.

Un feto pequeño para la edad gestacional es aquel cuyo peso fetal está entre el percentil 3 y 10, muestra una valoración anatómica por ultrasonido normal, presenta pruebas de bienestar fetal normales y al realizar una valoración prospectiva persiste en similares percentiles de crecimiento. ${ }^{1-7,16}$

La historia clínica aislada subestima el diagnóstico en un tercio de los casos de RCIU. ${ }^{7,14}$ En el examen físico se emplea la medición de la altura uterina. Dicha medición, aunque presenta sensibilidades que varían de $20 \%$ a $50 \%$, es fácil de hacer, no es costosa y debe realizarse en toda gestante como prueba primaria de tamizaje para detectar alteraciones en el crecimiento del feto. ${ }^{1-7,17,18}$ Una medida de tres o cuatro centímetros por debajo de lo esperado para la edad gestacional obliga a descartar la RCIU y amerita una valoración mediante ultrasonido (nivel de evidencia II y III, recomendación tipo C). ${ }^{18}$ De este modo, la opinión de este consenso es que las personas que realicen un ultrasonido obstétrico (obstetras y radiólogos) posean un entrenamiento adecuado y certificado, pues éste constituye el pilar principal para el diagnóstico (recomendación tipo D).

Además, siempre se debe tener en cuenta la fecha de la última menstruación o el ultrasonido del primer trimestre con el fin de asignar percentiles de crecimiento al feto. Los parámetros a valorar por ultrasonido son: $:^{1-7,17,19,20}$ 
- Cálculo del peso fetal: se recomienda la fórmula de Hadlock que utiliza la medición del perímetro cefálico, la circunferencia abdominal y la longitud del fémur. Tiene un error estimado de $+/-15 \%$ del peso real.

- Cálculo del percentil de crecimiento: después de estimar el peso fetal y conociendo la edad gestacional se calcula el percentil de crecimiento. Al comparar los valores obtenidos del peso fetal por ultrasonido con las curvas de Hadlock, se asignan los percentiles correspondientes a cada medición.

- Valoración del volumen de líquido amniótico: los valores del índice de líquido amniótico (ILA) inferiores o iguales a $5 \mathrm{~cm}$ se considerarán como "oligoamnios", entre $5 \mathrm{~cm}-7 \mathrm{~cm}$ como "límite inferior de la normalidad" y valores entre $8 \mathrm{~cm}-18 \mathrm{~cm}$ como "líquido amniótico normal". Las personas que no tienen un entrenamiento adecuado en la valoración del índice de líquido amniótico en los cuatro cuadrantes realizan falsamente diagnósticos de oligoamnios mientras que algunos autores prefieren la realización de la columna máxima, considerando oligoamnios exclusivamente cuando no existe una ventana de líquido amniótico superior a $2 \mathrm{~cm} .^{2,5,19,21-24}$

- Seguimiento longitudinal del feto y evaluación de la velocidad de crecimiento (en los casos en que sea posible): cuando no se cuenta con una edad gestacional por datos de ultrasonido temprano ni por amenorrea, es aconsejable realizar un ultrasonido con intervalos de dos semanas para poder establecer la tendencia de crecimiento. Se recomienda este tiempo, pues en un período mas breve resulta imposible diferenciar si los cambios observados en la medición se deben a la variación inter o intraobservador o a los cambios en el crecimiento fetal. . $^{2,417-20}$

Cuando en una valoración ultrasonográfica se detecta un crecimiento inferior al percentil 10 o existe sospecha de RCIU, se debe remitir a la paciente a una unidad de medicina maternofetal o especialista en perinatología para una valoración integral de la madre y su feto que incluya: valoración morfológica fetal, búsqueda de signos ecográficos asociados con enfermedad cromosómica y/o signos de infección y pruebas de bienestar fetal (nivel de evidencia III, recomendación tipo C). Es indispensable además, practicar pruebas de bienestar fetal que contengan al menos la valoración del Doppler de la arteria umbilical, de la arteria cerebral media y de las arterias uterinas ${ }^{24-36}$ (niveles de evidencia I,II y III; recomendaciones tipo A, B y C). Asimismo, se debe realizar una búsqueda del factor desencadenante y ofrecer estudios genéticos y/o estudios para infección de acuerdo con los hallazgos encontrados por medio del ultrasonido. Los fetos con malformaciones y con restricción del crecimiento intrauterino serán considerados como RCIU, pero su manejo dependerá de las anomalías asociadas.

\section{ETIOLOGÍA}

La RCIU y el feto PEG no constituyen una entidad específica y son una manifestación de enfermedades fetales, maternas o placentarias ${ }^{1-7}$ o simplemente una variación estadística poblacional de la normalidad. La presencia de uno o más de los factores patológicos descritos aumentan la probabilidad diagnóstica de la RCIU. ${ }^{17-19}$

- Causas maternas: deprivación nutricional materna severa, enfermedades vasculares (hipertensión, diabetes pregestacional, preeclampsia), enfermedades cardíacas, enfermedades respiratorias, renales y trombofilias; ingestión de algunos medicamentos; el abuso del alcohol, tabaco o drogas psicoactivas y antecedente de feto pequeño en embarazos anteriores.

- Causas fetales: constitucionales (feto constitucionalmente pequeño), anormalidades cromosómicas, malformaciones estructurales, infecciones fetales, displasias esqueléticas y la gestación múltiple. 
- Causas placentarias: insuficiencia placentaria, infartos placentarios, vasculitis, placenta previa, corioamnionitis crónica, inserción anormal del cordón umbilical, placenta circunvalada, anormalidades placentarias en la gestación múltiple, corioangioma y arteria umbilical única.

\section{PREVENCIÓN PRIMARIA}

\section{DE LA RCIU}

Aunque para algunos autores el control prenatal es cuestionable en cuanto a la prevención de la RCIU, ${ }^{37-}$

${ }^{39}$ la modificación del estilo de vida, la disminución de las carencias nutricionales y el abandono del consumo de tabaco, alcohol y drogas psicotrópicas son medidas generales que pueden impactar en el peso fetal ${ }^{5,7}$ (niveles de evidencia II y III, recomendaciones tipo $\mathrm{C}$ y D).

Una de las recomendaciones de este consenso es que, en lo posible, toda mujer que aspire a quedar embarazada (particularmente si presenta antecedentes de RCIU severa lejos del término, preeclampsia, cardiopatías, enfermedades renales o del colágeno y trombofilias) debería ser valorada preconcepcionalmente por un obstetra especialista en medicina materno-fetal (perinatología) o en su defecto por un obstetra con experiencia en alto riesgo obstétrico (nivel de evidencia IV, recomendación tipo D). Aunque no se ha demostrado clínicamente con respecto a la prevención de la $\mathrm{RCIU},{ }^{37-41}$ es prioritario en el control prenatal de todas las mujeres en embarazo la educación sobre conductas saludables, tales como la nutrición, la prevención de ITS y el evitar la exposición a agentes medio ambientales potencialmente nocivos para el embarazo y el feto (nivel de evidencia IV, recomendación tipo D).

\section{PREVENCIÓN SECUNDARIA DE LA RCIU}

En pacientes con factores de riesgo establecido, principalmente con antecedentes de preeclampsia severa lejos del término, trombofilias y enfermedades del colágeno, se ha demostrado que la utilización de ácido acetilsalicílico (ASA, por sus siglas en inglés) 100 mg día permite mejorar el resultado perinatal y disminuir las tasas de preeclampsia, aunque no se ha demostrado que reduzca la incidencia de bajo peso al nacer ${ }^{41-48}$ (nivel de evidencia $\mathrm{Ib}$, recomendación tipo A).

En estas pacientes de alto riesgo, la utilización del Doppler de arterias uterinas en la semana 12-14 o en la semana 20-24 de gestación, permite identificar el subgrupo de pacientes que van a desarrollar una RCIU más severa y precoz y/o preeclampsia. Es una recomendación de este consenso que en pacientes con factores de riesgo se evalúen las arterias uterinas mediante estudio Doppler. Una vez detectada la alteración de las arterias uterinas, la utilización de ASA en la semana 11-14 parece disminuir la frecuencia de bajo peso y preeclampsia, efecto que no se ha demostrado en pacientes en quienes se detecta la alteración en las arterias uterinas en la semana 20-24 y se administra el mismo medicamento ${ }^{43-45,49,50}$ (nivel de evidencia II, recomendación tipo B).

En los últimos años, la combinación de marcadores bioquímicos (proteína específica de la placenta PAPP-A y hormona gonadotropina humana fracción libre BHCG libre, etc.) con factores de riesgo, y el Doppler de las arterias uterinas en el primer trimestre como predictor de preeclampsia y RCIU han recibido una considerable atención. Aunque es susceptible de aplicar en la práctica clínica de nuestro medio, faltan estudios prospectivos que establezcan su validez. ${ }^{51,52}$

\section{PREVENCIÓN TERCIARIA EN LA RCIU}

Una vez establecido el diagnóstico de la RCIU, se debería efectuar una valoración integral en un tercer nivel de atención que cuente con personal obstétrico y neonatal bien entrenado. Se debe realizar una correcta clasificación, búsqueda de las enfermedades maternas o fetales subyacentes y hacer las pruebas de bienestar fetal adecuadas, para definir el diagnóstico y el momento óptimo para la finalización de la gestación. Esta conducta es la única que ha demostrado incidir sobre la mortalidad perinatal y la morbilidad a largo plazo, $7,16,53$ (nivel de evidencia II, recomendación tipo B). 
La utilización de medidas como: reposo absoluto, hiperoxigenación materna, agonistas, B-adrenérgicos, nifedipina, heparina y anticoagulantes, suplementos nutricionales y expansores del plasma no ha demostrado un efecto beneficioso sobre la RCIU establecida y, por lo tanto, no están recomendados ${ }^{54-56}$ (nivel de evidencia Ib, recomendación tipo A).

\section{CONTROL DEL FETO CON SOSPECHA DE RCIU}

El feto pequeño para la edad gestacional se diferencia del feto con verdadera RCIU por las ecografías de seguimiento y el Doppler de arterias uterinas y el Doppler fetal. El feto con crecimiento entre los percentiles 3-10, con pruebas de bienestar fetal normales, líquido amniótico en cantidad adecuada, Doppler fetal y de arterias uterinas normales se catalogará como "feto pequeño para la edad gestacional" y se recomendará seguimiento ecográfico de crecimiento y Doppler cada 2 semanas. ${ }^{1-7,16,21-26}$ Estos fetos no se benefician de la inducción electiva del parto antes de la semana 40.

\section{Valoración por estudios Doppler materno-fetales de la circulación placentaria:}

Los fetos con RCIU secundaria a insuficiencia placentaria, los cuales constituyen el grupo de más alto riesgo, presentan cambios progresivos de aumento de la resistencia placentaria, hipoxia y sospecha de asfixia, que pueden ser detectados mediante Doppler, perfil biofísico y monitoreo fetal electrónico (NST). ${ }^{24-36}$

Aunque la aproximación Doppler de múltiples vasos es lo ideal, deben tenerse en cuenta otros parámetros para finalizar la gestación, tales como el perfil biofísico fetal y el monitoreo fetal anteparto. ${ }^{57-62}$ En los embarazos complicados con RCIU secundario a insuficiencia placentaria se sugiere realizar un enfoque de valoración de múltiples vasos que incluya: arteria umbilical, arteria cerebral media, arterias uterinas y en casos más severos: ductus venoso, vena umbilical, istmo aórtico y posiblemente parámetros de función cardíaca fetal.
En feto con alteración hemodinámica grave, lejos del término, un estudio no logró demostrar que una conducta agresiva de finalización inmediata podría mejorar el pronóstico perinatal. Por tal motivo, la aproximación en estos casos, particularmente en los de lejos del término, debe ser individualizada. ${ }^{63}$ Entre las pruebas de bienestar fetal, el estudio Doppler de la arteria umbilical ha demostrado reconocer los fetos con crecimiento restringido que requieren mayor atención y vigilancia más estrecha; por lo tanto, una de las recomendaciones de este panel de consenso es que se involucre el estudio Doppler en el feto con sospecha de RCIU (nivel de evidencia $\mathrm{Ib}$, recomendación tipo A).

Además, no existen tablas en nuestro medio y con nuestra población para saber cuáles son los valores de Doppler que superan el parámetro de la normalidad. Ante la ausencia de estos datos, el mencionado consenso sugiere que éste debería ser un campo de investigación; y por lo tanto, se adaptarán las tablas de estudios de otras poblaciones, con el objetivo de definir los parámetros de anormalidad. Asimismo, se considera que los índices de pulsatilidad (IP) y la morfología de las ondas Doppler, son los parámetros más reproducibles. Por dicha razón, el estudio Doppler debe ser realizado por un personal capacitado y un equipo idóneo. Los reportes generados de estudios Doppler deben contener la valoración cualitativa de la onda y una valoración cuantitativa (IP). Se sugiere adoptar las siguientes tablas de referencia:

- Relación cerebro-placentaria: Baschat A. UOG $2003 .{ }^{64}$

- Índice de pulsatilidad en la arteria umbilical: Kiserud 2006. ${ }^{65,66}$

- Doppler de arterias uterinas: Gratacos et al 2007.67

- Ductus venoso: Kiserud 2006. ${ }^{68}$

- Flujo en el istmo aórtico: Fouron 2003. ${ }^{69-70}$

De acuerdo con la literatura revisada, este consenso considera que la secuencia de los cambios Doppler debe establecerse bien y su informe debe ser estandarizado, por tal motivo éstos se describen en la tabla 2. 
Tabla 2. Secuencia de la velocimetría Doppler en fetos afectos por RCIU.

\section{Cambios iniciales en el Doppler:}

- Aumento de la resistencia en las arterias uterinas: presencia de Notch (escotadura) bilateral o índice de pulsatilidad (IP) promedio de las dos arterias superior al percentil 95.

- Aumento en el IP de la arteria umbilical (superior al percentil 95 para la edad gestacional), con presencia de diástole umbilical y sin cambios en la circulación cerebral.

En estos casos, el informe ecográfico-Doppler dirá: "feto con aumento de las resistencias placentarias sin signos de redistribución hemodinámica".

\section{Cambios del Doppler sugestivos de hipoxia fetal:}

- Alteración de la relación cerebro-placentaria.

- Vasodilatación de la arteria cerebral media (ACM), con un IP de la ACM inferior al percentil 5.

- Aumento en el IP de la arteria umbilical con ausencia de flujo en diástole.

En los casos anteriores es obligatorio realizar la valoración de los vasos venosos, ductus venoso y vena umbilical, y el flujo en estos vasos debe ser normal.

El informe del ultrasonido dirá: "feto con signos de redistribución hemodinámica".

\section{Cambios Doppler de posible asfixia fetal:}

- Flujo diastólico reverso en la arteria umbilical.

- Ausencia de flujo atrial en el ductus venoso.

- Presencia de onda "a" reversa en el ductus venoso.

- Presencia de flujo venoso pulsátil en la vena umbilical periférica o intraabdominal.

Al realizar el informe se catalogará como: "feto con alteración hemodinámica grave”. En estos casos, se recomienda realizar una valoración funcional cardíaca fetal, con énfasis en la relación e/a de las válvulas tricúspide y mitral, valoración de existencia de regurgitación tricúspide, el índice TEI y valoración cualitativa y cuantitativa del istmo aórtico. Todo lo anterior con el objetivo de individualizar cada caso, particularmente en fetos pretérminos.

\section{SEGUIMIENTO}

Dado que el feto con RCIU está en riesgo de muerte y de sufrir morbilidad, se debe realizar una vigilancia fetal cuidadosa que incluya el Doppler y las otras pruebas de bienestar fetal como el monitoreo fetal y el perfil biofísico. ${ }^{57-62}$ La severidad de la enfermedad y su progresión generalmente dependen de la edad de detección de la RCIU y de la severidad de la enfermedad placentaria (cambios en la arteria umbilical).

Entre los "criterios de severidad" en las pruebas de bienestar fetal se consideran los siguientes:

- IP en el ductus venoso superior al percentil 95.

- Signos Doppler sugestivos de posible "asfixia fetal".

- Perfil biofísico fetal menor o igual a 6/10 con oligoamnios.

- Prueba no estresante (NST) con variabilidad menor a 5 latidos y presencia de desaceleraciones.
La actuación del obstetra para decidir el momento de la terminación de la gestación debe radicar en sopesar entre los riesgos de la prematuridad y los riesgos de dejar el feto en un ambiente hostil, que pueden llevar a complicaciones a corto y largo plazo. $^{1-7}$

De otra parte, no existe evidencia de que el manejo hospitalario de la madre se asocie con mejores resultados perinatales, ni con un mayor peso neonatal; sin embargo, parece aconsejable recomendar reposo y eliminar posibles factores externos desencadenantes. Por lo tanto, es necesaria la hospitalización en los siguientes casos: presencia de síndrome hipertensivo asociado con el embarazo, cambios Doppler sugestivos de asfixia fetal, enfermedades maternas asociadas, dificultad para realizar un adecuado seguimiento ambulatorio o 
indicación de terminación de la gestación ${ }^{61-65}$ (nivel de evidencia IV, recomendación tipo D).

Los criterios para la inducción de la maduración fetal con esteroides no cambian con respecto a las normas generales (24-34 semanas) y se deben utilizar cuando se piensa finalizar la gestación entre los rangos establecidos ${ }^{16,71}$ (nivel de evidencia Ia, recomendación tipo A).

En la tabla 3 se presenta el resumen de las recomendaciones de este consenso para el manejo, vía del parto y monitorización en diferentes escenarios clínicos de fetos con RCIU ${ }^{1-7,16,57-63,72-74}$ (niveles de evidencia II, III y IV, recomendaciones B, C y D).

\section{SITUACIONES ESPECIALES}

- Estancamiento de crecimiento fetal: se define como la ausencia de crecimiento fetal por ecografía en un intervalo de dos o más semanas. Este hallazgo amerita un seguimiento estricto con pruebas de bienestar fetal y, si el feto ya tiene maduración pulmonar comprobada mediante conteo de cuerpos lamelares o pruebas similares, se puede considerar la terminación de la gestación ${ }^{3-7,16,62}$ (nivel de evidencia IV, recomendación tipo D).

- En pacientes con preeclampsia o trastornos hipertensivos, los cambios Doppler y las pruebas de vigilancia fetal pierden valor predictivo negativo, por lo tanto se recomienda una vigilancia fetal más estricta (nivel de evidencia IV, recomendación tipo D).

- Se desaconseja la útero-inhibición en pacientes con síntomas de parto pretérmino y que presenten RCIU severa de origen placentario, pues estos síntomas pueden ser reflejo de un abruptio inicial o de una descompensación fetal ${ }^{1-7}$ (nivel de evidencia III, recomendación tipo C).

- En pacientes con embarazo múltiple esta guía de vigilancia se puede aplicar para las gestaciones bicoriales. En gestaciones monocoriales y en el contexto de la RCIU, este protocolo de vigilancia pierde validez, particularmente por la presencia de conexiones arterio-arteriales (niveles de evidencia III y IV).
- En gestaciones cercanas al término (superior a 35 semanas) puede existir vasodilatación cerebral (IP < percentil 5) o alteración de la relación cerebro-placentaria, sin cambios en la arteria umbilical. Estos hallazgos se deben corroborar con una nueva medición en 12-24 horas y otras pruebas de bienestar fetal. En caso de que estas pruebas de bienestar se encuentren alteradas y de que persista la vasodilatación, se considerará finalizar la gestación $^{3-7,62}$ (niveles de evidencia III y IV).

- Los fetos afectados por RCIU, particularmente los que presenten alteraciones en el Doppler, presentan un riesgo elevado de morbi-mortalidad perinatal, por tal motivo se considera que debe existir disponibilidad de cuidado intensivo neonatal con personal entrenado ${ }^{16,75-78}$ (nivel de evidencia IV, recomendación tipo D).

\section{SEGUIMIENTO POSNATAL}

Se considera indispensable la valoración mediante anatomía patológica de la placenta en los casos de RCIU. ${ }^{79}$ Con respecto a la madre, la presencia de RCIU severa en forma precoz debe llevar a una valoración materna en el posparto de los posibles factores desencadenantes como son: síndrome de anticuerpos antifosfolípidos, enfermedades del colágeno, nefropatías y trombofilias. Es aconsejable una visita al especialista en medicina maternofetal o alto riesgo obstétrico, para la adecuada consejería y valoración. En estos casos, se desaconseja la utilización de anticonceptivos orales hasta completar el estudio ${ }^{1-7}$ (nivel de evidencia IV, recomendación tipo D).

Los niños propensos a la RCIU deben ser evaluados durante intervalos regulares, una vez superado el período posnatal, en búsqueda de alteraciones del desarrollo psicomotor. En la vida adulta, se han encontrado alteraciones tales como diabetes, hipertensión y síndrome metabólico; por lo tanto, debería existir una intervención sobre los factores de riesgo cardiovasculares ${ }^{76-78,80-82}$ (nivel de evidencia IV, recomendación tipo D). 
Tabla 3. Resumen de las recomendaciones para el manejo, vía del parto y monitorización

de diferentes escenarios clínicos de fetos con RCIU (niveles de evidencia II, III y IV, recomendaciones

$$
\text { tipos B, C y D). }{ }^{1-7,16,57-63,72-75}
$$

\section{$R C I U$ sin signos sugestivos de insuficiencia placentaria (Doppler normal):}

- Hospitalización: no requerida.

- Seguimiento eco/Doppler: semanal.

- Control bienestar fetal: según edad gestacional, pero se recomienda NST y perfil biofísico semanal.

- Terminación de la gestación: ante presencia de criterios de severidad en las pruebas de bienestar fetal o a las 37 semanas.

- Vía del parto: de acuerdo con criterios obstétricos. Se debe realizar vigilancia fetal intraparto con auscultación intermitente como está indicada para embarazos de alto riesgo y según la Organización Mundial de la Salud (OMS): fase latente, cada 30 minutos; trabajo de parto activo, cada 15 minutos; expulsivo, cada 5 minutos.

$R C I U$ con "aumento de la resistencia placentaria pero sin signos de redistribución hemodinámica” y flujo diastólico presente en la arteria umbilical:

- Hospitalización: no imprescindible.

- Seguimiento eco/Doppler: semanal.

- Control bienestar fetal: NST y perfil biofísico fetal semanal.

- Terminación de la gestación: ante la presencia de criterios de severidad en las pruebas de bienestar fetal o a las 37 semanas.

- Vía del parto: de acuerdo con criterios obstétricos. Requiere vigilancia fetal intraparto con auscultación intermitente para embarazo de alto riesgo según OMS y/o monitoreo electrónico continuo o intermitente (niveles de evidencia Ia y III).

\section{RCIU con "aumento de la resistencia placentaria y con signos de redistribución hemodinámica":}

- Hospitalización: individualizar, se recomienda vigilancia más estrecha.

- $\quad$ Seguimiento eco/Doppler: cada 48-72 horas.

- Control bienestar fetal: NST y PBF cada 72 horas.

- Terminación de la gestación: ante presencia de 'criterios de severidad' en las pruebas de bienestar fetal o a la semana 34 (nivel de evidencia III).

- Vía del parto: de acuerdo con criterios obstétricos. Requiere vigilancia fetal intraparto con auscultación intermitente para embarazo de alto riesgo según Organización Mundial de la Salud (OMS) y/o monitoreo electrónico continuo o intermitente. Si existe dificultad para la monitorización considerar el parto por cesárea.

\section{RCIU con "aumento de la resistencia placentaria y alteración hemodinámica grave":}

- Se considera que el feto presenta un estado de descompensación grave y puede morir en días. Monitorización estricta.

- Hospitalización: requerida.

- $\quad$ Seguimiento eco/Doppler: diario.

- Control bienestar fetal: NST y PBF diario.

- Terminación de la gestación:

- Gestación mayor de 34 semanas: terminación inmediata.

- Gestación de 28-34 semanas: maduración pulmonar y terminación a criterio médico, teniendo en cuenta la disponibilidad de camas en la UCI neonatal y los resultados de las pruebas de bienestar fetal. Se recomienda tener en cuenta los parámetros de función cardíaca fetal.

- Gestación menor de 28 semanas: analizar viabilidad fetal de acuerdo con el peso fetal calculado y los resultados perinatales de la UCI neonatal. Informar a los padres de los riesgos y los beneficios. Suministrar esteroides. Si el ductus venoso conserva la onda "a" positiva atrial se puede considerar esperar plazos cortos. Se recomienda tener en cuenta los parámetros de función cardíaca fetal. En estos casos existe alta probabilidad de muerte neonatal, complicaciones y secuelas (niveles de evidencia III y IV).

- Vía del parto: se recomienda la cesárea en los casos en que se considere que el feto es viable (nivel de evidencia III). 


\section{Consenso Nacional de Expertos. Medellín (Colombia), septiembre de 2008.}

- José Enrique Sanín-Blair, M.D. Gineco-obstetra. Coordinador del Consenso Colombiano de RCIU. Especialista en Medicina Materno-Fetal. Clínica Universitaria Bolivariana, Especialista en Ultrasonido. Medicina Fetal S.A, Medellín (Colombia). Correo electrónico: saninblair@yahoo.com.

- Jaime Gómez Díaz, M.D. Gineco-obstetra. Especialista en Ultrasonido. Medicina Fetal S.A, Medellín (Colombia).

- Jorge Ramírez, M.D. Gineco-obstetra. Especialista en Ultrasonido. Medicina Fetal S.A, Medellín (Colombia).

- Carlos Alberto Mejía, M.D. Gineco-obstetra. Especialista en Ultrasonido. Medicina Fetal S.A, Medellín (Colombia).

- Óscar Medina, M.D. Gineco-obstetra. Especialista en Ultrasonido. Medicina Fetal S.A, Medellín (Colombia).

- José Vélez, M.D. Gineco-obstetra. Especialista en Ultrasonido. Medicina Fetal S.A, Medellín (Colombia).

- Carlos Restrepo, M.D. Gineco-obstetra. Especialista en Ultrasonido. Medicina Fetal S.A, Medellín (Colombia).

- Jorge Gutiérrez, M.D. Gineco-obstetra. Especialista en Medicina Materno-Fetal. Clínica Universitaria Bolivariana. Especialista en Fetología. Clínica del Prado, Medellín (Colombia).

- Arturo Cardona, M.D. Gineco-obstetra. Especialista en Fetología. Clínica del Prado, Medellín (Colombia).

- Felipe Jaramillo-Daza, M.D. Gineco-obstetra. Especialista en Medicina Materno-Fetal. Universidad CES. Hospital General de Medellín, Medellín (Colombia).

- Marco Duque, M.D. Gineco-obstetra. Especialista en Medicina Materno-Fetal. Fundación Universitaria Sanitas y Clínica de la Mujer, Bogotá (Colombia).

- Andrés Sarmiento, M.D. Gineco-obstetra. Coordinador de Ginecología y Obstetricia. Fundación Santa Fe. Bogotá (Colombia).

- Julián Delgado, M.D. Gineco-obstetra. Especialista en Medicina Materno-Fetal. Hospital Universitario del Valle. Cali (Colombia).

- Jaime López-Tenorio, M.D. Gineco-Obstetra. Especialista en Medicina Materno Fetal. Hospital Valle de Lili. Cali (Colombia).

- Mónica Beltrán, M.D. Gineco-obstetra. Especialista en Medicina Materno-Fetal. Universidad Industrial de Santander. Bucaramanga (Colombia).

- Carlos Becerra, M.D. Gineco-obstetra. Especialista en Medicina Materno-Fetal. Universidad Industrial de Santander. Bucaramanga (Colombia).

- Libardo Gómez, M.D. Gineco-obstetra. Especialista en Perinatología. CEDIUL. Barranquilla (Colombia).

- Nelson Aguilar, M.D. Gineco-obstetra. Epidemiólogo. Presidente de FECOPEN. Medellín (Colombia).

- Óscar Ordóñez, M.D. Gineco-obstetra. Universidad del Cauca. Popayán (Colombia).

- Gustavo Vásquez, M.D. Gineco-Obstetra. Especialista en Perinatología. Universidad Libre de Cali. Cali (Colombia).

- Francisco Salcedo, M.D. Gineco-obstetra. Universidad de Cartagena. Cartagena (Colombia).
- Carlos Echeverri, M.D. Gineco-obstetra. Especialista en Medicina Materno-Fetal. Pereira (Colombia).

- Édgar Acuña, M.D. Gineco-obstetra. Especialista en Medicina Materno-Fetal. Fundación Universitaria San José. Bogotá (Colombia).

\section{REFERENCIAS}

1. Ville Y, Nyberg DA. Growth, Doppler and fetal assessment. En: Nyberg DA, McGahan JP, Pretorius DH, Pilu G, editors. Diagnostic imaging of fetal anomalies. Philadelphia: Lippincott Williams \& Wilkins; 2003. p. 31-58.

2. Manning FA. General principles and applications of ultrasonography. En: Creasy RK, Resnik R, editors. Maternal-fetal medicine. 4th ed. Philadelphia: W.B. Saunders Company; 1999. p. 169-206.

3. Figueras F, Gratacos E. Maneig de les alteracions per defecte del creixement fetal. Servicio de Medicina Materno Fetal. Institut Clínic de Ginecologia, Obstetrícia i Neonatología (ICGON), Hospital Clínic de Barcelona; 2007.

4. Sanin-Blair JE, Cuartas A. Restricción del crecimiento intrauterino. En: Botero J, Jubiz A, Henao G, eds. Obstetricia y Ginecología-Texto integrado. 8a ed. Bogotá: Editorial Québec World; 2008. p. 242-44.

5. ACOG practice bulletin No. 12. Washington, DC: American College of Obstetricians and Gynecologists; 2000.

6. Gratacós E, Carreras E, Higueras T, Becker J, Delgado J, López J, et al. Protocolo de incorporación del Doppler en el diagnóstico y control del feto RCIU o bajo peso; 2003.

7. Baschat A. Fetal Growth disorders. En: James DK, Steer PJ, Weiner CP, Gonik B. High risk obstetrics: management options. 3rd ed. Elsevier editorial; 2005. p. 240-72.

8. Departamento Administrativo Nacional de Estadística - DANE. Visitado en 2009 Jul 16. Disponible en http://www.dane.gov.co

9. Sacket DL. Evidence-based Medicine: How to Practice and Teach EBM. 2nd ed. New York: Churchill Livingstone; 2000. p. 1-252.

10. Hadlock FP, Harris RB, Sharman RS, Deter RL, Park SK. Estimation of fetal weight with the use of head, body and femur measurements-a prospective study. Am J Obstet Gynecol 1985;151:333-7.

11. Montoya-Restrepo NE, Correa-Morales JC. Curvas de peso al nacer. Rev Salud Pública 2007;9:1-10. 
12. Lagos R, Espinoza R, Echeverría P, Graf D, Sepúlveda JD, Orellana JJ. Gráfica regional de crecimiento fetal normal. Rev Hosp Mat Inf Ramón Sardá 2002;21:3-10.

13. González R, Gómez R, Castro R, Nien JK, Merino P, Etchegaray A, et al. Curva nacional de distribución de peso al nacer según edad gestacional: Chile, 1993 a 2000. Rev Med Chile 2004;132:1155-65.

14. Espinoza R, Lagos R, Guerra F. Guía para restricción de crecimiento intrauterino; 2003.

15. Boletín CLAP 19/94: Normatización de ecografías obstétricas. Montevideo, Uruguay; 1994.

16. Royal College of Obstetricians and Gynaecologists. The investigation and management of the small for gestational age; 2002.

17. Platz E, Newman R. Diagnosis of IUGR: traditional biometry. Semin Perinatol 2008;32:140-7.

18. Neilson JP. Symphysis-fundal height measurement in pregnancy. Cochrane Database Syst Rev 2000;(2): CD000944.

19. Baschat AA. Fetal responses to placental insufficiency: an update. BJOG 2004;111:1031-41.

20. Baschat AA. Pathophysiology of fetal growth restriction: implications for diagnosis and surveillance. Obstet Gynecol Surv 2004;59:617-27.

21. Resnik R. Intrauterine Growth Restriction. Obstet Gynecol 2002;99:490-6.

22. Turan S, Miller J, Baschat AA. Integrated testing and management in fetal growth restriction. Semin Perinatol 2008;32:194-200.

23. Nabhan AF, Abdelmoula YA. Amniotic fluid index versus single deepest vertical pocket as a screening test for preventing adverse pregnancy outcome. Cochrane Database Syst Rev 2008;(3):CD006593.

24. Lalor JG, Fawole B, Alfirevic Z, Devane D. Biophysical profile for fetal assessment in high risk pregnancies. Cochrane Database Syst Rev 2008; (1): CD000038.

25. Nielson JP, Alfirevic Z. Doppler ultrasound for fetal assessment in high risk pregnancies. Cochrane Database Syst Rev 2000;(2):CD000073.

26. Maulik D. Doppler Ultrasound in Obstetrics and Gynecology. 2nd ed. Heidelberg, Germany: Springer; 2005.
27. Mari G, Picconi J. Doppler vascular changes in intrauterine growth restriction. Semin Perinatol 2008;32:182-9.

28. Harman CR, Baschat AA. Comprehensive assessment of fetal wellbeing: which Doppler tests should be performed? Curr Opin Obstet Gynecol 2003; 15:147-57.

29. Sebire NJ. Umbilical artery Doppler revisited: pathophysiology of changes in intrauterine growth restriction revealed. Ultrasound Obstet Gynecol 2003;21:419-22.

30. Baschat AA, Gembruch U, Weiner CP, Harman CR. Qualitative venous Doppler waveform analysis improves prediction of critical perinatal outcomes in premature growth-restricted fetuses. Ultrasound Obstet Gynecol 2003;22:240-5.

31. Baschat AA, Weiner C. Umbilical artery Doppler screening for detection of the small fetus in need of antepartum surveillance. Am J Obstet Gynecol 2000;182:154-8.

32. Hecher K, Bilardo CM, Stigter RH, Ville Y, Hackelöer BJ, Kok HJ, et al. Monitoring of fetuses with intrauterine growth restriction: a longitudinal study. Ultrasound Obstet Gynecol 2001;18:564-70.

33. Baschat AA, Gembruch U, Harman CR. The sequence of changes in Doppler and biophysical parameters as severe fetal growth restriction worsens. Ultrasound Obstet Gynecol 2001;18:571-7.

34. Miller J, Turan S, Baschat AA. Fetal growth restriction. Semin Perinatol 2008;32:274-80.

35. Turan OM, Turan S, Gungor S, Berg C, Moyano D, Gembruch U, et al. Progression of Doppler abnormalities in intrauterine growth restriction. Ultrasound Obstet Gynecol 2008;32:160-7.

36. Turan S, Turan OM, Berg C, Moyano D, Bhide A, Bower $\mathrm{S}$, et al. Computerized fetal heart rate analysis, Doppler ultrasound and biophysical profile score in the prediction of acid-base status of growth-restricted fetuses. Ultrasound Obstet Gynecol 2007;30:750-6.

37. Coria-soto IL, Bobadilla JL, Notzon F. The effectiveness of antenatal care in preventing intrauterine growth retardation and low birth weight due to preterm delivery. Int J Qual Health Care 1996;8:13-20. 
38. Lu MC, Tache V, Alexander GR, Kotelchuck M, Halfon N. Preventing low birth weight: is prenatal care the answer? J Matern Fetal Neonatal Med 2003;13:362-80.

39. Villar J, Merialdi M, Gülmezoglu AM, Abalos E, Carroli G, Kulier R, de Onis M. Characteristics of randomized controlled trials included in systematic reviews of nutritional interventions reporting maternal morbidity, mortality, preterm delivery, intrauterine growth restriction and small for gestational age and birth weight outcomes. J Nutr 2003;133:1632S-1639S.

40. de Onis M, Villar J, Gülmezoglu M. Nutritional interventions to prevent intrauterine growth retardation: evidence from randomized controlled trials. Eur J Clin Nutr 1998;52:S83-93.

41. Gülmezoglu M, de Onis M, Villar J. Effectiveness of interventions to prevent or treat impaired fetal growth. Obstet Gynecol Surv 1997;52:139-48.

42. Trudinger BJ, Cook CM, Thompson RS, Giles WB, Connelly A. Low-dose aspirin therapy improves fetal weight in umbilical placental insufficiency. Am J Obstet Gynecol 1988;159:681-5.

43. CLASP: a randomised trial of low-dose aspirin for the prevention and treatment of pre-eclampsia among 9364 pregnant women. CLASP (Collaborative Lowdose Aspirin Study in Pregnancy) Collaborative Group. Lancet 1994;343:619-29.

44. Leitich H, Egarter C, Husslein P, Kaider A, Schemper M. A meta-analysis of low dose aspirin for the prevention of intrauterine growth retardation. $\mathrm{Br}$ J Obstet Gynaecol 1997;104:450-9.

45. Italian study of aspirin in pregnancy. Low-dose aspirin in prevention and treatment of intrauterine growth retardation and pregnancy-induced hypertension. Lancet 1993;341:396-400.

46. Harrington K, Kurdi W, Aquilina J, England P, Campbell S. A prospective management study of slow-release aspirin in the palliation of uteroplacental insufficiency predicted by uterine artery Doppler at 20 weeks. Ultrasound Obstet Gynecol 2000;15:13-8.

47. Yu CK, Papageorghiou AT, Parra M, Dias RP, Nicolaides $\mathrm{KH}$. Randomized controlled trial using low-dose aspirin in the prevention of pre-eclampsia in women with abnormal uterine artery Doppler at 23 weeks' gestation. Ultrasound Obstet Gynecol 2003;22:233-9.

48. Coomarasamy A, Papaioannou S, Gee H, Khan KS. Aspirin for the prevention of preeclampsia in women with abnormal uterine artery Doppler: a metaanalysis. Obstet Gynecol 2001;98:861-6.

49. Vainio M, Kujansuu E, Iso-Mustajärvi $M$, Mäenpää J. Low dose acetylsalicylic acid in prevention of pregnancy-induced hypertension and intrauterine growth retardation in women with bilateral uterine artery notches. BJOG 2002;109:161-7.

50. Ebrashy A, Ibrahim M, Marzook A, Yousef D. Usefulness of aspirin therapy in high-risk pregnant women with abnormal uterine artery Doppler ultrasound at 14-16 weeks pregnancy: randomized controlled clinical trial. Croat Med J 2005;46:826-31.

51. Gagnon A, Wilson RD, Audibert F, Allen VM, Blight C, Brock JA, et al. Obstetrical complications associated with abnormal maternal serum markers analytes. J Obstet Gynaecol Can 2008;30:918-32.

52. Pihl K, Larsen T, Krebs L, Christiansen M. First trimester maternal serum PAPP-A, beta-hCG and ADAM12 in prediction of small-for-gestational-age fetuses. Prenat Diagn 2008;28:1131-5.

53. Mari G, Hanif F, Treadwell MC, Kruger M. Gestational age at delivery and Doppler waveforms in very preterm intrauterine growth-restricted fetuses as predictors of perinatal mortality. J Ultrasound Med 2007;26:555-9.

54. Say L, Gülmezoglu AM, Hofmeyr GJ. Maternal nutrient supplementation for suspected impaired fetal growth. Cochrane Database Syst Rev 2003;(1): CD000148.

55. Say L, Gülmezoglu AM, Hofmeyr GJ. Maternal oxygen administration for suspected impaired fetal growth. Cochrane Database Syst Rev 2003;(1):CD000137.

56. Say L, Gülmezoglu AM, Hofmeyr GJ. Hormones for suspected impaired fetal growth. Cochrane Database Syst Rev 2003;(1):CD000109.

57. Baschat AA, Galan HL, Bhide A, Berg C, Kush ML, Oepkes D, et al. Doppler and biophysical assessment in growth restricted fetuses: distribution of test results. Ultrasound Obstet Gynecol 2006;27:41-7. 
58. ACOG Practice Bulletin. Clinical Management Guidelines for Obstetrician-Gynecologists, Number 70, December 2005 (Replaces Practice Bulletin Number 62, May 2005). Intrapartum fetal heart rate monitoring. Obstet Gynecol 2005; 106:1453-60.

59. Devoe L, Golde S, Kilman Y, Morton D, Shea K, Waller J. A comparison of visual analyses of intrapartum fetal heart rate tracings according to the new National Institute of Child Health and Human Development guidelines with computer analyses by an automated fetal heart rate monitoring system. Am J Obstet Gynecol 2000;183:361-6.

60. Pardey J, Moulden M, Redman CW. A computer system for the numerical analysis of nonstress tests. Am J Obstet Gynecol 2002;186:1095-103.

61. Cosmi E, Ambrosini G, D’Antona D, Saccardi C, Mari G. Doppler, cardiotocography, and biophysical profile changes in growth-restricted fetuses. Obstet Gynecol 2005;106:1240-5.

62. Baschat AA. Integrated fetal testing in growth restriction: combining multivessel Doppler and biophysical parameters. Ultrasound Obstet Gynecol 2003;21:1-8.

63. Thornton JG, Hornbuckle J, Vail A, Spiegelhalter DJ, Levene M. Infant wellbeing at 2 years of age in the Growth Restriction Intervention Trial (GRIT): multicentred randomised controlled trial. Lancet 2004;364:513-20.

64. Baschat AA, Gembruch U. The cerebroplacental Doppler ratio revisited. Ultrasound Obstet Gynecol 2003;21:124-7.

65. Acharya G, Wilsgaard T, Berntsen GK, Maltau JM, Kiserud T. Reference ranges for serial measurements of blood velocity and pulsatility index at the intraabdominal portion, and fetal and placental ends of the umbilical artery. Ultrasound Obstet Gynecol 2005;26:162-9.

66. Acharya G, Wilsgaard T, Berntsen GK, Maltau JM, Kiserud T. Reference ranges for serial measurements of umbilical artery Doppler indices in the second half of pregnancy. Am J Obstet Gynecol 2005;192:937-44.

67. Gómez O, Figueras F, Fernández S, Bennasar M, Martínez JM, Puerto B, et al. Reference ranges for uterine artery mean pulsatility index at 11-41 weeks of gestation. Ultrasound Obstet Gynecol 2008;32:128-32.

68. Kessler J, Rasmussen S, Hanson M, Kiserud T. Longitudinal reference ranges for ductus venosus flow velocities and waveform indices. Ultrasound Obstet Gynecol 2006;28:890-8.

69. Fouron JC. The unrecognized physiological and clinical significance of the fetal aortic isthmus. Ultrasound Obstet Gynecol 2003;22:441-7.

70. Ruskamp J, Fouron JC, Gosselin J, Raboisson MJ, Infante-Rivard C, Proulx F. Reference values for an index of fetal aortic isthmus blood flow during the second half of pregnancy. Ultrasound Obstet Gynecol 2003;21:441-4.

71. Bernstein IM, Horbar JD, Badger GJ, Ohlsson A, Golan A. Morbidity and mortality among very-lowbirth-weight neonates with intrauterine growth restriction. The Vermont Oxford Network. Am J Obstet Gynecol 2000;182:198-206.

72. Baschat AA, Cosmi E, Bilardo CM, Wolf H, Berg C, Rigano S, et al. Predictors of neonatal outcome in early-onset placental dysfunction. Obstet Gynecol 2007;109:253-61.

73. Mari G, Hanif F, Kruger M. Sequence of cardiovascular changes in IUGR in pregnancies with and without preeclampsia. Prenat Diagn 2008;28:377-83.

74. Mari G, Hanif F, Kruger M, Cosmi E, SantolayaForgas J, Treadwell MC. Middle cerebral artery peak systolic velocity: a new Doppler parameter in the assessment of IUGR fetuses. Ultrasound Obstet Gynecol 2007;29:310-6.

75. Confidential Enquiry into Stillbirths and Deaths in Infancy. Annual Report for 1 January - 31 December 1993. London: HMSO; 1996.

76. Spinillo A, Capuzzo E, Stronati M, Ometto A, De Santolo A, Acciano S. Obstetric risk factors for periventricular leukomalacia among preterm infants. Br J Obstet Gynaecol 1998;105:865-71.

77. Amato M, Konrad D, Hüppi P, Donati F. Impact of prematurity and intrauterine growth retardation on neonatal hemorrhagic and ischemic brain damage. Eur Neurol 1993;33:299-303. 
78. Thornton JG, Hornbuckle J, Vail A, Spiegelhalter DJ, Levene M; The GRIT study Group. Infant wellbeing at 2 years of age in the Growth Restriction Intervention Trial (GRIT): multicentred randomized controlled trial. Lancet 2004;364:513-20.

79. Tyson RW, Staat BC. The Intrauterine growthrestricted fetus and placenta evaluation. Semin Perinatol 2008;32:166-71.
80. Hales CN, Barker DJ. The thrifty phenotype hypothesis. Br Med Bull 2001;60:5-20.

81. Singhal A, Lucas A. Early origins of cardiovascular disease: is there a unifying hypothesis? Lancet 2004;363:1642-5.

82. Hofman PL, Regan F, Jackson WE, Jefferies C, Knight DB, Robinson EM, et al. Premature birth and later insulin resistance. N Engl J Med 2004;351:2179-86.

Conflicto de intereses: ninguno declarado.

Fuente de financiación: este consenso fue realizado con el auspicio de FECOPEN (Federación Colombiana de Perinatología) y Medicina Fetal S.A. de Medellín (Colombia). No se recibieron aportes de casas farmacéuticas o casas comerciales para la elaboración de esta guía. 